\title{
SISTEMAS DE INCENTIVOS GERENCIAIS E O RISCO DAS EMPRESAS DO ESTADO DO ESPÍRITO SANTO
}

\section{SYSTEMS OF MANAGERIAL INCENTIVE AND THE RISK OF FIRMS IN THE STATE OF ESPIRITO SANTO}

\section{SISTEMAS DE INCENTIVOS GERENCIALES Y EL RIESGO DE LAS EMPRESAS DEL ESTADO DE ESPÍRITO SANTO}

\section{ARIDELMO JOSÉ CAMPANHARO TEIXEIRA}

Contador. Doutor em Controladoria e Contabilidade pela USP. Professor da Fucape Business School. Coordenador do Programa de Pós-Graduação Stricto Sensu (Mestrado e Doutorado) em Ciências Contábeis e Administração da Fucape Business School. Endereço: Av. Fernando Ferrari, 1358. 29075 505. Boa Vista - Vitória-ES CEP 29075-505. E-mail: aridelmo@fucape.br

\section{GRAZIELA XAVIER FORTUNATO}

Engenheira de produção. Doutora em Administração de Empresas pela PUC-Rio. Mestrado em Administração Financeira pelo IBMEC. Professora da Fucape Business School. Consultora na área de Gestão de empresas. Endereço: Av. Fernando Ferrari, 1358. 29075505.

Boa Vista - Vitória-ES CEP 29075-505. E-mail: grazielafortunato@fucape.br

\section{ARILDA MAGNA CAMPAGNARO TEIXEIRA}

Economista. Doutora em Economia pela UFRJ. Professora da Fucape Business School. Coordenadora Executiva da Graduação da Fucape Business School. Endereço: Av. Fernando Ferrari, 1358. 29075 505. Boa Vista - Vitória-ES CEP 29075-505. E-mail: arildat@fucape.br

\section{KADIJA CELANTE PIZETA}

Administradora. Mestranda em Administração de Empresas pela Fucape Business School. Endereço: Av. Fernando Ferrari, 1358. 29075 505. Boa Vista - Vitória-ES CEP 29075-505.

E-mail:kadijag@hotmail.com 


\section{RESUMO}

O estudo objetivou verificar a relação entre o nível risco da empresa e os incentivos gerenciais oferecidos aos gestores de tais empresas. Essa ideia foi baseada na Teoria de Agência que prevê que as empresas, ao oferecerem sistemas de incentivos aos gestores, tendem a alcançar o alinhamento de interesses entre os agentes. Dessa forma, é esperado que os gestores não assumam riscos em níveis acima dos requeridos pelos acionistas. Portanto, os riscos atribuídos às empresas estariam associados à presença de incentivos oferecidos aos gestores. Essa é uma análise empírica com amostra composta pelas empresas presentes na classificação das 200 maiores empresas do Estado do Espírito Santo, publicada pela revista Findes, edição de 2008. A coleta de dados ocorreu no período de maio a dezembro de 2009 , por meio de entrevista pessoal in loco, com aplicação de questionário semiestruturado para identificar as empresas que aplicavam sistemas de incentivo e para extrair as variáveis aplicadas no modelo de regressão logística. Os resultados encontrados levantam evidências de que não há relação entre o nível de risco das empresas e os sistemas de incentivos oferecidos aos gestores.

Palavras-Chave: Conflito de Interesse; Remuneração Variável; Teoria de Agência.

\section{ABSTRACT}

This study aimed to verify the relation between the level of risk the firm undergoes and managerial incentives offered to the managers of said firms. This idea was based on the Agency Theory that predicts that the firms, when offering systems of managerial incentives, tend achieve an alignment of interests between agents. In this manner, it is expected that the managers do not take higher level risk than those accepted by stockholders. Therefore, risks attributed to the firms would be associated with the presence of the incentives offered to their managers. This is an empirical analysis with a sample composed by the firms ranked among the 200 largest firms of the State of Espírito Santo, released by a regional magazine Findes, in 2008. The data were collection occurred during the period of May to December 2009, through interviews in loco, and with the application of a semi-structured questionnaire to identify the firms that adopted systems of managerial incentive, and to extract the variables applied to the logistic regression model. The acquired results provided evidence that there is no relation between the level of risk the firms take and the systems incentive offered to their managers.

Keywords: Conflict of Interest; Variable Income; Agency Theory. 


\section{repc}

\section{RESUMEN}

El estudio objetivó verificar la relación entre el nivel de riesgo de la empresa y los incentivos gerenciales ofrecidos a los gestores de tales empresas. Esa idea fue basada en la Teoría de Agencia que prevé que las empresas, al ofrecer sistemas de incentivos a los gestores, tienden a alcanzar el alineamiento de intereses entre los agentes. De esa forma, es esperado que los gestores no asuman riesgos en niveles por encima de los requeridos por los accionistas. Por tanto, los riesgos atribuidos a las empresas estarían asociados a la presencia de incentivos ofrecidos a los gestores. Ése es un análisis empírico con muestra compuesta por las empresas presentes en la clasificación de las 200 mayores empresas del Estado de Espírito Santo, publicado por la revista Findes edición de 2008. La colecta de datos ocurrió en el período de mayo a diciembre de 2009, por medio de entrevista personal in loco, con aplicación de cuestionario semi-estructurado para identificar a las empresas que aplicaban sistemas de incentivo y para extraer las variables aplicadas en el modelo de regresión logística. Los resultados encontrados levantan evidencias de que no hay relación entre el nivel de riesgo de las empresas y los sistemas de incentivos ofrecidos a los gestores.

Palabras Clave: Conflicto de Interés; Remuneración Variable; Teoría de Agencia.

\section{INTRODUÇÃO}

Empresas de todos os setores têm atrelado suas operações ao risco que estas oferecem, sendo que este não pode ser eliminado completamente, cabendo apenas estratégias para seu gerenciamento. O risco atrelado às operações refere-se às perdas resultantes da utilização de sistemas, modelos de gestão, controles inadequados, interesses divergentes, bem como falha humana (JORION, 1997).

Podem ocorrer em alguns casos que os interesses dos gestores sejam distintos dos interesses da empresa, influenciando assim os riscos em função da não congruência de objetivos. Uma das formas utilizadas pelas empresas para alinhamento de interesses entre os gestores e proprietários do capital, ou seja, os agentes, é oferecer sistemas de incentivos que não encorajam os gestores a assumirem risco excessivo. Os sistemas de incentivos gerenciais oferecidos aos gestores exercem papel motivador que induz o comportamento dos agentes, para que estes e a organização alcancem o alinhamento de objetivos (BAILEY; BROWN; COCCO, 1998; MERCHANT; VAN DER STEDE; ZHENG, 2003).

Segundo Fessler (2003), os estudos sobre sistemas de incentivos oferecidos aos gestores, especificamente os monetários, buscam identificar comportamentos disfuncionais; problemas com a utilização de medidas contábeis inadequadas; aspectos motivacio- 
nais e outros fatores que possam influenciar a obtenção da congruência de objetivos. Os comportamentos disfuncionais ocorrem quando não há conformidade entre as metas das organizações e gestores, levando-os a assumir risco excessivo, em níveis distintos dos requeridos pela organização.

Para Horngren, Sundem e Stratton (2004), os incentivos oferecidos aos gestores podem exercer o papel de influenciar as decisões gerenciais, a fim de que não seja assumido risco excessivo pelo gestor e não prejudique a organização. Ademais, quando há incentivos oferecidos aos gestores, os mesmos irão maximizar o resultado para influenciar sua remuneração. Portanto, se por um lado os interesses dos gestores e da organização podem ser alinhados, utilizando sistemas de incentivo (MERCHANT; VAN DER STEDE; ZHENG, 2003), por outro, os gestores podem não assumir risco e prejudicar a organização (OYADOMARI et al., 2008). Basu (1997) ressalta que, se porventura, os gestores tiverem a possibilidade de gerenciar o resultado da empresa, eles o farão de forma a se beneficiar.

Diante do exposto, faz-se o seguinte questionamento: há relação entre os sistemas de incentivos gerenciais oferecidos aos gestores e o risco das empresas?

Para responder a esse questionamento, este trabalho tem como objetivo verificar a relação entre a presença de incentivos gerenciais oferecidos aos gestores e o risco atribuído às organizações. A análise dessa relação é baseada na ideia de que empresas que apresentam risco tendem a possuir sistemas de controle. Com isso, espera-se encontrar relação positiva entre o risco das empresas e a sua adoção por sistemas de controle. A amostra foi composta por 140 empresas presentes na classificação das 200 maiores empresas do Espírito Santo, publicada pela revista Findes de 2008, cuja coleta de dados ocorreu por meio de entrevista pessoal in loco com aplicação de questionário semiestruturado. A metodologia inclui o modelo de regressão logística, pelo fato da variável dependente, risco, ser dicotômica. A proxy utilizada para risco é a ocorrência de prejuízo líquido do exercício.

Segundo Murphy (1999), os sistemas de incentivos, ou tipo de remuneração dos gestores, é um assunto que vem despertando interesse da comunidade científica nos últimos anos. Aguiar et al. (2009), partindo do princípio de controlabilidade, investigaram a relação entre certas práticas de Contabilidade com a presença de sistema de incentivo gerencial, com resultados indicando que práticas como centro de responsabilidade, orçamento e avaliação de desempenho parecem servir de base para a estruturação de tais sistemas de incentivo, não sendo possível dizer o mesmo em relação às práticas de custo-padrão e preço de transferência. Na mesma linha, Dalmacio, Rezende e Slomski (2009) verificaram a variabilidade de alguns indicadores contábeis utilizados como medida de performance nos contratos de remuneração variável dos gestores com o intuito de alinhar interesses e minimizar custos de agência. Seus resultados mostram que o setor é o direcionador da 
utilização desses indicadores como parâmetros de remuneração dos gestores. Teixeira et al. (2011), referindo-se não a sistema de incentivo gerencial, mas a sistemas de controle gerencial, analisaram a relação destes com fatores organizacionais.

No entanto, dada a dificuldade de acesso às informações, o assunto ainda se encontra incipiente e sugere aprofundamento. Assim, como principal contribuição, o artigo oferece à pesquisa em Contabilidade Gerencial um novo olhar sobre o problema de agência, bem como o avanço do tema.

\section{REFERENCIAL TEÓRICO}

\subsection{Sistemas de Incentivos Gerenciais}

Sistemas de incentivos gerenciais são oferecidos aos gestores com algumas finalidades, como por exemplo, alinhamento de interesses entre os diversos agentes e a organização (ANTHONY; GOVINDARAJAN, 2008); aplicação como ferramenta gerencial para motivar ou induzir o comportamento dos gestores para garantir o alcance dos objetivos da organização (BAILEY; BROW; COCCO, 1998; MERCHANT; VAN DER STEDE; ZHENG, 2003). No entanto, gestores tendem a disponibilizar maior quantidade de esforços em atividades remuneradas.

Anthony e Govindarajan (2008) dividem incentivos gerenciais como positivos e negativos, sendo esses caracterizados pelas recompensas e punições, respectivamente. Se por um lado incentivo gerencial positivo ou recompensa provoca no gestor aumento de satisfação, por outro, a recompensa negativa ou punição reduz a satisfação. Ainda segundo Anthony e Govindarajan (2008), o gestor se motiva mais quando induzido por recompensa do que pelo medo de punição.

Além disso, o gestor pode ser incentivado tanto com remuneração quanto com incentivos não financeiros. Os incentivos financeiros são importantes, porém o reconhecimento do seu desempenho como profissional também o motiva na realização das tarefas, emergindo assim a necessidade de sincronização dos incentivos oferecidos (ANTHONY; GOVINDARAJAN, 2008; HORNGREN; SUNDEM; STRATTON, 2004).

Incentivos financeiros incluem aumentos de salários, bônus, benefícios e prerrogativas (automóveis, viagens de férias e associação em clubes) e incentivos não financeiros incluem possibilidade de promoção, aumento de responsabilidade, maior autonomia, melhor localização geográfica e reconhecimento. Para Bonner e Sprinkle (2002), incentivos financeiros podem provocar um aumento do esforço gerencial que poderia afetar positivamente o desempenho das organizações.

Neste sentido, Aguiar et al. (2009); Bailey, Brow e Cocco (1998); Baker, Jensen e Murphy (1988); Melumad, Mookherjee e Reichelstein (1992) corroboram com Boner e Sprinkle (2002) ao afirmar que incentivos financeiros ainda podem influenciar o compor- 
tamento dos gestores de modo a garantir determinado nível de desempenho e evitar que esses assumam riscos excessivos, divergindo dos acionistas.

Basu (1997) tem uma visão negativa dos sistemas de incentivo que se justifica pelo fato de considerar que se o gestor tiver a sua remuneração ligada aos resultados e o mesmo puder influenciá-los, ele o fará de modo a se beneficiar.

Ademais, sistemas de incentivos gerenciais teriam a função de alinhamento de interesses entre gestores e acionistas (INDJEJIKIAN; NANDA, 1999). Assim, espera-se que os sistemas de incentivos gerenciais, por meio da função de criação da congruência de objetivos, evitem que os gestores incorram em riscos maiores que os requeridos pelas empresas, uma vez que o risco poderá reduzir sua base de cálculo de ganho, criando comportamentos disfuncionais como manipulação de resultados, criação de reservas e miopia gerencial (AGUIAR et al., 2009; HIRST, 1983; MERCHANT; VAN DER STEDE; ZHENG, 2003).

\subsection{Risco Empresarial}

As empresas, em busca do cumprimento de suas missões, expõem-se aos riscos em suas operações, requerendo planejamento e monitoramento dos mesmos.

Gitman (1997) define risco como a possibilidade de prejuízo financeiro ou, mais formalmente, classifica o termo risco como incerteza ao referir-se à variabilidade de retornos associados a um ativo. Ainda segundo Gitman (1997), os ativos expostos a maiores possibilidades de perdas são vistos como mais arriscados na comparação com aqueles que possuem menores possibilidades de perdas.

Enquanto Gitman (1997) classifica risco como incerteza, há divergências sobre essa afirmativa. Em geral, os termos risco e incerteza são usados simultaneamente para se referir à variabilidade dos retornos esperados. No entanto, os estatísticos definem risco e incerteza pelo conhecimento da probabilidade de ocorrerem possíveis resultados, e assim, legitima-se a diferença entre os termos. Em seu artigo seminal de 1921, Frank Knight define essa distinção claramente ao se referir a risco como aos eventos sujeitos ao conhecimento de sua distribuição de probabilidade e incerteza como aqueles sobre os quais não é possível especificar a probabilidade numericamente. Simplificadamente, o risco existe quando a distribuição de probabilidade dos resultados possíveis é conhecida, e a incerteza existe quando a distribuição de probabilidade não é conhecida (LEROY e SINGELL JR., 1987). Werlang (1998) explica que para evitar confusões semânticas, este conceito é chamado de incerteza knightiana e em modelos nos quais a incerteza reduz-se a risco, chama-se de bayesiano.

Ross, Westerfield e Jaffe (1995, p.232) elucidam que o verdadeiro risco de qualquer investimento é representado pela parcela inesperada do retorno, resultante de surpresas que podem advir durante o processo. O risco pode ser de diversas categorias, tais como: mercado, crédito, câmbio, operacionais e legais; contudo, inerente às operações se encontram o risco operacional. 
Segundo Deloach (2001, p.226), o risco operacional representa a chance de que as operações sejam ineficientes ou ineficazes ao executar o modelo de negócios da empresa, ao satisfazer as necessidades dos clientes, ao atender a requisitos de qualidade, custo e desempenho. Saunders (2000, p.104-105) relata que o risco operacional pode surgir sempre que a tecnologia existente deixar de funcionar adequadamente ou quando os sistemas de apoio falhar.

Duarte $\mathrm{Jr}$ (2001) divide o risco em três categorias: i) risco organizacional que ocorre quando a organização se mostra ineficiente; a gestão se torna inconsistente e sem objetivos de longo prazo definidos; o fluxo de informações é fraco; as responsabilidades são mal definidas e há ocorrência de fraudes e acesso a informações internas por parte dos concorrentes; ii) risco operacional que está relacionado a falhas de sistemas, tais como, telefônico, elétrico, computacional, de processamento e de armazenamento de dados passíveis de fraudes e erros; iii) risco de pessoal que ocorre quando há ausência de alinhamento de objetivos entre a empresa, funcionários, gestores e demais stakeholders.

A utilidade da segregação dos riscos está em conseguir informações sobre a contribuição de cada tipo para o resultado final da empresa, segundo Bergamini Jr. (2005). Ainda conforme o autor, o reconhecimento de que os riscos são multidimensionais emergiu com o progressivo conhecimento das especificidades do risco operacional. Com base nessa constatação, os riscos foram segregados, e mesmo que se defina ou se categorize os tipos de riscos, não sendo este um consenso na literatura, o objetivo é o olhar holístico e a gestão integrada destes (BERGAMINI JR., 2005).

As falhas humanas que afetam o funcionamento do sistema ou mesmo a rentabilidade da empresa podem estar associadas às falhas de gerenciamento. Se o gerente não é motivado a realizar tarefas com grandes probabilidades de riscos e não for reconhecido quando a operação é concluída com sucesso, a propensão é ele não as assumir. Contudo, levar os gestores a assumirem riscos de fatores incontroláveis pode resultar em um custo alto para as empresas, provocando assim o surgimento de mecanismos de alinhamento entre interesses de gestores e acionistas (AGUIAR et al., 2009; MERCHANT; VAN DER STEDE; ZHENG, 2003).

Segundo Basu (1997), o gestor tem influência direta nos resultados da empresa e, se porventura, tiver seus vencimentos vinculados ao desempenho, suas ações irão corroborar com a proposição de Jensen e Meckling (1976) de que este, de fato, influenciará no resultado em busca de satisfação pessoal, que neste caso, pode ser a remuneração.

\subsection{Teoria de Agência}

A separação entre propriedade e controle nas corporações modernas tem influenciado as questões acerca da Teoria de Agência identificada por Berle e Means (1932); Jensen e Meckling (1976); Murphy (1999). A discussão relacionada aos problemas de incentivos 


\section{repc}

Aridelmo José Campanharo Teixeira, Contador. Doutor em Controladoria, Graziela Xavier Fortunato, Arilda Magna Campagnaro Teixeira e Kadija Celante Pizeta

gerenciais e avaliação de desempenho e baseada na Teoria de Agência é direcionadora de pesquisas em Contabilidade Gerencial (BANKER; DATAR, 1989; FELTHAM, XIE, 1994; DIKOLLI, 2001; DIKOLLI; VAYSMAN, 2006; HEMMER, 1996; MURPHY, 1999).

A Teoria de Agência concentra-se no problema da ausência de alinhamento de interesses entre os agentes ou no relacionamento estabelecido entre dois ou mais indivíduos que possa afetar o desempenho organizacional (BERLE; MEANS, 1932).

A Teoria de Agência fundamenta-se na relação entre agente e principal, na qual o principal não tendo conhecimento total sobre determinado assunto, ou não possuindo disponibilidade de tempo para realização das tarefas requeridas, delega e remunera um agente para executá-las (GIETZMANN, 1995; JENSEN; MECKLING, 1976). Nesse contexto, o principal, na figura de proprietário do capital, delega funções aos agentes ou gestores e espera que esses atuem com os interesses alinhados aos seus. Porém, há possibilidade dos agentes agirem de maneira a maximizar seus próprios interesses em detrimento aos interesses do principal, ocorrendo assim desalinhamento de objetivos (CARDOSO; MARIO; AQUINO, 2007; JENSEN; MURPHY; WRUCK, 2004).

Para garantir que os gestores tomem decisões e assumam riscos, conforme requeridos pelo principal, esses oferecem sistemas de incentivos com a função contratual de alinhamento de interesses (GIETZMANN, 1995). Segundo Camargos, Helal e Boas (2007), problemas previstos pela teoria de agência têm sido solucionados em algumas organizações, atrelando a remuneração dos gestores ao desempenho financeiro da empresa, por meio dos incentivos oferecidos.

Dessa forma, a Teoria de Agência estabelece as relações de trabalho entre seus participantes por meio de contratos nos quais aspectos relacionados à conduta e remuneração são regulamentados e explicitados (COSENZA; ALEGRÍA; LAURENCEL, 2009).

Nessa visão, a organização é vista como um nexo de contratos estabelecidos entre as partes integrantes. Para Consenza, Alegria e Laurencel (2009) e Lambert (2001), sem o monitoramento dos contratos, o agente pode usar da assimetria de informação para expropriar o principal sem colocar em risco seus incentivos. Nesse caso, ocorrem ainda situações nas quais o gestor, sabendo que suas ações não são conhecidas plenamente pelo principal, utiliza-se de recursos da organização com propósitos distintos do principal, de forma que esse recurso não impacte sua remuneração. Em função dessa falta de alinhamento de interesses, a empresa pode sair prejudicada em função da assimetria de informação, ou seja, o gestor possui informações sobre suas ações que o principal pode desconhecer (CARDOSO; MARIO; AQUINO, 2007).

Dado que os acionistas ou o principal sempre estarão interessados nos resultados gerados pelos gestores, a Teoria da Agência fornece estrutura para o papel desempenhado pela Contabilidade no fornecimento de informações para avaliação, monitoramento e criação de mecanismos de incentivos. Dessa forma, os gestores estão aptos a oferecer aos 
acionistas informações gerenciais sobre a organização, reduzindo a assimetria informacional (HENDRIKSEN; VAN BREDA, 1999).

Com isso, é possível observar que os elementos fundamentais incluídos na Teoria da Agência estão relacionados à existência de assimetria de informação, de riscos assumidos e de sistemas de incentivos. Problemas com sistemas de incentivos podem ocorrer em função da falta de congruência de objetivos e da existência de problemas quando o gestor expõe a organização a um risco acima do requerido pelo principal (COSENZA; ALEGRÍA; LAURENCEL, 2009).

\section{METODOLOGIA}

Para atingir o objetivo de verificar a relação entre a presença de incentivos gerenciais oferecidos aos gestores e o risco atribuído às organizações, este trabalho se baseia em pesquisa empírico-analítica com a utilização de questionário como instrumento de coleta de dados, contendo 44 questões abertas e fechadas.

Antes da aplicação do questionário à totalidade da amostra, houve um pré-teste, em abril de 2009, com 13 empresas escolhidas por acessibilidade com o objetivo de detectar os pontos fracos desse instrumento, além de conferir maior confiança e precisão aos resultados (COOPER e SCHINDLER, 2003, p. 83). Não houve a necessidade de incluir ou excluir nenhuma questão. O questionário foi montado com perguntas objetivas, a fim de verificar se as empresas aplicam sistemas incentivos gerenciais. Não houve a necessidade de elaborar um construto, tampouco realizar análise de confiabilidade e validade.

Com isso, a coleta de dados ocorreu por meio de entrevista pessoal in loco. Após a validação do questionário, as empresas eram contatadas por meio telefônico para a apresentação dos propósitos da pesquisa e a realização de agendamento da visita. As visitas para coleta de dados ocorreram entre o período de maio a dezembro de 2009. Dessas questões aplicadas, foram extraídas as variáveis utilizadas no teste estatístico desta pesquisa, que foram: risco assumido, setor econômico de atuação da empresa e número de empregados para representar o tamanho, e se a empresa oferece ou não incentivos.

\subsection{Amostra}

A amostra analisada é não probabilística e essa escolha ocorreu pelo critério de acessibilidade e disponibilidade dos dados por parte das mesmas. Foram enviados os questionários para as 200 maiores empresas do Estado do Espírito Santo, classificadas pela revista Findes, edição 2008. Dos 200 questionários enviados, 140 foram devolvidos com as respostas, portanto, a amostra totaliza 140 empresas. Os respondentes foram classificados pelas empresas como gestores ou responsáveis pela tomada de decisões. As principais características dos respondentes descritas na Tabela 1. 


\section{TABELA 1: CARACTERÍSTICAS DOS RESPONDENTES}

\begin{tabular}{|c|c|c|c|c|c|}
\hline \multicolumn{3}{|c|}{ Cargo Ocupado } & \multicolumn{3}{|c|}{ Tempo no Cargo Ocupado } \\
\hline Presidente & 1 & $1 \%$ & Menos de 5 anos & 68 & $49 \%$ \\
\hline Diretor & 21 & $15 \%$ & Entre 5 a 10 anos & 32 & $23 \%$ \\
\hline Gerente & 70 & $50 \%$ & Entre 10 a 15 anos & 20 & $14 \%$ \\
\hline Coordenador & 26 & $19 \%$ & Mais de 15 anos & 20 & $14 \%$ \\
\hline Outros & 22 & $16 \%$ & & & \\
\hline Total & 140 & $100 \%$ & Total & 140 & $100 \%$ \\
\hline
\end{tabular}

Fonte: Elaborado pelos autores.

Na Tabela 1, pode-se observar que a maioria dos respondentes ocupa cargo de gerente $(50 \%)$, seguidos por coordenadores (19\%), diretores $(15 \%)$ e presidente $(1 \%)$. Outros cargos ocupados representam $16 \%$ da amostra. A pesquisa apresentou um índice de $49 \%$ para profissionais com menos de 5 anos no cargo, seguido de $23 \%$ entre 5 a 10 anos, $14 \%$ entre 10 e 15 anos e $14 \%$ com mais de 15 anos.

\subsection{Modelo e Variáveis Utilizadas}

Para alcançar o objetivo proposto, utilizou-se o método de regressão logística. A escolha dessa ferramenta ocorreu em função da variável dependente ser binária e da possibilidade de realização de inferências quanto à probabilidade de associação entre as variáveis (HAIR et al., 2005).

Para verificar a possível relação entre o risco e a presença de sistemas de incentivos nas empresas da amostra, utilizou-se como variável dependente o risco, cuja proxy adotada foi o lucro líquido. Se determinada empresa obteve lucro, isso indica que houve entrada e saída de recursos, gerando resultado positivo, assim, o risco dessa empresa pode ser considerado menor do que as empresas que demonstraram resultado negativo de suas operações (BEATTY; ZAJAC, 1994). Ressalta-se que o lucro ou o prejuízo líquidos foram utilizados como medidas de risco em outros trabalhos, como o de Altman (1968), o de Beaver (1966) e o de Zmijewski (1984). A variável risco assume valor zero (0) quando a empresa apresenta lucro e assume valor um (1) quando a empresa apresenta prejuízo.

Com relação aos sistemas de incentivos, foi questionado se as empresas ofereciam algum sistema de incentivos aos gestores. Baseado nas respostas dos gestores, foi atribuído valor zero (0) para as empresas que afirmaram não oferecer sistemas de incentivo e valor um (1) para as empresas que afirmaram que ofereciam algum sistema de incentivo 
aos gestores. Segundo Beatty e Zajac (1994), existe uma relação inversa entre o nível de incentivos gerenciais oferecidos aos gestores e o risco atribuído à empresa. Ainda segundo os autores, as empresas utilizam os sistemas de incentivos para minimizar os conflitos de agência e reduzir o risco atribuído às mesmas. Ademais, os incentivos oferecidos aos gestores fazem parte das ferramentas da Contabilidade gerencial utilizadas pelas empresas para profissionalização da gestão e consequentemente, minimização dos riscos (DAVILA; FOSTER, 2007).

Duas variáveis de controle foram utilizadas no modelo proposto, tais como: tamanho e setor econômico. A variável tamanho, tendo como proxy o número de empregados (MERCHANT, 1990; DAVILA; FOSTER, 2007), foi empregada em função dos incentivos gerenciais serem utilizados, principalmente por grandes empresas com capital pulverizado, como forma de alinhamento de interesse e minimização dos possível conflitos de agência que poderiam surgir (CAMARGOS; HELAL; BOAS, 2007). A variável setor econômico foi utilizada em função de alguns setores apresentarem características particulares que possam influenciar os níveis de riscos das empresas. Dessa forma, assume valor 1 caso a empresa seja uma instituição financeira, valor 2 caso seja de utilidade pública, valor 3 para empresas comerciais, valor 4 para empresas do setor industrial e valor 5 para prestadores de serviços.

Utilizou-se o seguinte modelo de regressão logística (Equação 1) para verificação da possível associação entre as variáveis descritas:

$$
\text { Risco }_{i}=\beta_{1}+\beta_{2} \text { Incentivos }_{i}+\beta_{3} \text { Tamanho }^{i}+\beta_{4} \text { Setor }_{i}+e_{i}(1)
$$

Onde:

Risco $_{i}$ - Risco da empresa i, assumindo zero para resultado positivo e um para resultado negativo;

Incentivos - Sistemas de incentivos gerenciais oferecidos aos gestores, assumindo valor um para as empresas que oferecem sistemas de incentivos e zero para as que não oferecem;

Tamanho $_{i}$ - Número de empregados da empresa i;

Setor $_{i}$ - Setor de atuação da empresa i, assumido valor 1 para instituição financeira, 2 para empresa de utilidade pública, 3 para empresas comerciais, 4 para empresas industriais e 5 para prestadores de serviços.

\section{ANÁLISE DOS RESULTADOS}

Na Tabela 2, são apresentados os resultados da estatística descritiva das variáveis contínuas analisadas. 


\section{TABELA 2: ESTATÍSTICA DESCRITIVA VARIÁVEL CONTÍNUA - TAMANHO}

\begin{tabular}{|l|c|}
\hline \multicolumn{2}{|c|}{ Tamanho } \\
\hline $\mathrm{N}$ & 140 \\
\hline Média & 1894,67 \\
\hline Mediana & 300 \\
\hline Desvio - padrão & 8536,12 \\
\hline Mínimo & 0 \\
\hline Máximo & 88972 \\
\hline
\end{tabular}

Fonte: Elaborado pelos autores.

A Tabela 2 apresenta o resultado da estatística descritiva da variável tamanho, sendo essa, a única variável contínua dentre as variáveis analisadas. Na Tabela 3, são apresentados os resultados da estatística descritiva das variáveis categóricas.

\section{TABELA 3: ESTATÍSTICA DESCRITIVA DAS VARIÁVEIS CATEGÓRICAS}

\begin{tabular}{|c|c|c|c|c|c|c|c|c|}
\hline Risco & Qtde. & $\%$ & Incentivo & Qtde. & $\%$ & Setor & Qtde. & $\%$ \\
\hline $\begin{array}{l}\text { Resultado } \\
\text { Positivo }\end{array}$ & 113 & $81 \%$ & Sim & 91 & $65 \%$ & Inst. Financeiras & 5 & $4 \%$ \\
\hline $\begin{array}{l}\text { Resultado } \\
\text { Negativo }\end{array}$ & 27 & $19 \%$ & Não & 49 & $35 \%$ & Ind. Ut. Pública & 1 & $1 \%$ \\
\hline \multirow[b]{4}{*}{ Total } & \multirow[b]{4}{*}{140} & \multirow[b]{4}{*}{$100 \%$} & \multirow[b]{4}{*}{ Total } & \multirow[b]{4}{*}{140} & \multirow[b]{4}{*}{$100 \%$} & Comercial & 39 & $28 \%$ \\
\hline & & & & & & Industrial & 54 & $39 \%$ \\
\hline & & & & & & Serviços & 41 & $29 \%$ \\
\hline & & & & & & Total & 140 & $100 \%$ \\
\hline
\end{tabular}

Fonte: Elaborado pelos autores.

As estatísticas descritivas das variáveis risco, incentivo e setor econômico foram realizadas com base na contagem das respostas. Utilizando o resultado líquido como proxy para a variável risco, é possível observar que a maioria das empresas da amostra (81\%) obtiveram resultado líquido positivo no período analisado. Quando questionadas sobre a presença de incentivos gerenciais, 65\% das empresas afirmaram oferecer sistemas de incentivos aos gestores, contrapondo-se a 35\% que afirmaram não oferecer qualquer tipo de incentivos aos gestores. Com relação ao setor econômico de atuação das empresas 
da amostra, há uma maior concentração entre os setores industrial, serviços e comercial, representando $39 \%$, $29 \%$ e $28 \%$, respectivamente.

Os resultados da regressão logística estão apresentados na Tabela 4:

\section{TABELA 4: ESTATÍSTICA DE REGRESSÃO LOGÍSTICA - EQUAÇÃO 1}

$$
\text { Risco }_{i}=\beta_{1}+\beta_{2} \text { Incentivos }_{i}+\beta 3 \text { Tamanho }_{i}+\beta_{4} \text { Setor }_{i}+\mathrm{e}
$$

\begin{tabular}{|l|c|c|c|}
\hline Sist. Incentivos & Odds Ratio & Estatística - Z & $\mathbf{P}$ - value \\
\hline Incentivos & 1,796264 & 1,20 & 0,229 \\
\hline Tamanho & 1,370164 & 1,64 & 0,102 \\
\hline Setor & 0,999886 & $-1,13$ & 0,256 \\
\hline No Observações: & & 140 \\
Wald chi2 & & 4,71 \\
Prob > chi2 & & 0,1944 \\
PseudoR2 & & 0,0320 \\
\hline
\end{tabular}

Fonte: Elaborado pelos autores.

Conforme resultados demonstrados na Tabela 4, verifica-se que não há associação entre as variáveis analisadas, pois nenhuma variável apresentou significância estatística ao nível de 10\%. Esse resultado levanta evidências de que não há relação entre o risco das empresas da amostra e o fato das mesmas oferecerem incentivos gerenciais aos gestores, considerando como proxy para risco o resultado líquido das empresas. Essa constatação diverge de algumas evidências empíricas encontradas na literatura (BEATTY; ZAJAC, 1994; MILLER; WISEMAN; GOMEZ-MEJIA, 2002). No entanto, concorda com os resultados de Teixeira et al, (2011), que não encontraram relação entre sistema de controle gerencial e o desempenho, utilizando como proxy o ROE. Isso mostra claramente que a dissonância dos resultados está relacionada com as amostras distintas. O estudo de Teixeira et al. (2011), adotou a mesma amostra do presente estudo.

Beatty e Zajac (1994), analisando uma amostra de empresas com Initial Public Offering (IPO), encontraram evidências de que há relação inversa entre os sistemas oferecidos aos gestores e as taxas de risco da amostra, utilizando como medida de risco o resultado líquido do período. Miller, Wiseman e Gomez-Mejia (2002) encontraram associação entre os riscos das empresas e a estrutura dos sistemas de incentivos oferecidos aos gestores.

Uma justificativa para os resultados encontrados divergirem do resultado de pesquisas anteriores de Baetty e Zajac (1994) e Miller, Wiseman e Gomez-Mejia (2002) pode ser a estrutura de propriedade. A maioria das empresas da amostra apresenta gestão familiar, 
apesar de algumas negociadas em bolsa também estarem presentes. Esse fato pode estar neutralizando o efeito do sistema de remuneração dos gestores.

Assim, a partir dos resultados encontrados, não é possível realizar inferências quanto à existência de relação entre os sistemas de incentivos oferecidos aos gestores e risco das empresas da amostra.

\section{CONSIDERAÇÕES FINAIS E SUGESTÃO PARA FUTURAS PESQUISAS}

Nesta pesquisa, buscou-se, por meio de um estudo empírico-analítico, verificar a existência de associação entre o risco das empresas da amostra e a presença de sistemas de incentivos oferecidos aos gestores. A amostra foi composta por 140 empresas, classificadas no ranking das 200 Maiores Empresas do Estado do Espírito Santo, sendo a pesquisa norteada pelos pressupostos da Teoria de Agência.

Neste estudo foram analisadas uma das questões centrais previstas pela Teoria de Agência, a de que os agentes possuem interesses e informações divergentes dos interesses do principal e não é possível assegurar que os gestores tomem as mesmas decisões que os acionistas tomariam (JENSEN; MURPHY; WRUCK, 2004). Ao oferecerem sistemas de incentivos aos gestores, as empresas esperam alcançar como resultado o alinhamento de interesses, de forma a influenciar o comportamento dos agentes para que não sejam assumidos riscos em níveis acima dos requeridos pelo principal.

Os resultados encontrados sugerem evidências de que não há associação entre os sistemas de incentivos oferecidos aos gestores e o risco atribuído às empresas analisadas, contrapondo a resultados encontrados anteriormente (BEATTY; ZAJAC, 1994; MILLER; WISEMAN; GOMEZ-MEJIA, 2002). Tal dissonância nos resultados é explicada pela diferença entre as amostras, dado que concorda com os resultados de Teixeira et al. (2010) cujos dados de amostra são similares.

Algumas implicações poderiam decorrer dos resultados encontrados. Primeiramente, caso a base para mensuração dos sistemas de incentivos esteja atrelada ao desempenho financeiro das empresas, pode-se esperar que os gestores não assumam riscos de fatores incontroláveis que poderiam influenciar em seus rendimentos. Contudo, as medidas utilizadas para avaliação de desempenho e a base para determinação de incentivos podem gerar alguns comportamentos disfuncionais não previstos, como: gerenciamento de resultados, criação de reservas e miopia gerencial (CHOW et al., 1996; MERCHANT, 1990; VAN DER STEDE, 2000). Futuras pesquisas nessa linha poderiam investigar a associação entre risco e sistemas de incentivos oferecidos aos gestores, levando em consideração a medida adotada pela empresa para avaliação do desempenho dos gestores.

A ausência de associação entre os riscos das empresas e os incentivos oferecidos pode ser influenciada pelo tipo de incentivo oferecido. Pesquisas futuras sobre incentivos poderiam analisar tal relação sob a perspectiva de tipos distintos de incentivos oferecidos 


\section{repc}

pelas empresas, como opções de ações, participação nos resultados e planos de incentivos de longo prazo.

A principal contribuição deste estudo foi oferecer evidências empíricas, utilizando dados coletados em campo, quanto à associação entre resultado das empresas e incentivos oferecidos aos gestores. Contudo, em suma, as evidências encontradas oferecem indícios contrários ao previsto pela Teoria da Agência, porém, o trabalho apresenta algumas limitações que carecem ser consideradas como existirem outras proxies que podem ser utilizadas para mensuração do risco, bem como outros fatores que podem influenciar a associação analisada, tais como: estrutura de capital da empresa e tempo do executivo no cargo. Ademais, os sistemas de incentivos oferecidos aos gestores podem ser utilizados com outras finalidades além do alinhamento de interesses entre agente e principal. Baseado nisso, não se pretende estabelecer nenhuma relação de causalidade, como a presença de incentivos iria dirimir os riscos da organização. Para tanto, seria necessária a utilização de outros métodos de pesquisa que possibilitassem isolar a influência de outros fatores distintos dos analisados.

Por fim, o presente estudo apresenta algumas limitações que necessitam serem consideradas. Uma limitação, por exemplo, é a amostra referir-se ao período de um ano gerando indisponibilidade de dados para verificar se os resultados persistiriam ao se considerar uma série temporal maior. Outra limitação desta pesquisa refere-se ao fato do estudo ter sido aplicado à maioria de empresas de capital fechado e o conflito entre agentes não ter sido captado, estatisticamente, devido ao cenário no qual as empresas estão inseridas, por exemplo, a gestão familiar, assim, os mecanismos de incentivo podem ter sua utilidade restrita

Assim, sugere-se em futuras pesquisas a ampliação do período de análise. Sugerese ainda, a utilização de outras medidas de desempenho como: Resultado/Lucro Líquido; Resultado/Lucro Operacional (EBITDA); faturamento; qualidade/satisfação do cliente; custos; volume de produção; market share. Recomenda-se também a inclusão da variável estrutura de propriedade como variável de controle, no sentido de controlar o efeito da gestão familiar nos resultados.

\section{REFERÊNCIAS BIBLIOGRÁFICAS}

AGUIAR, A.B.A.; TEIXEIRA, A.J.C.; NOSSA, V.; GONZAGA, R. P. Associação entre Sistemas de Incentivos Gerenciais e Utilização de Práticas de Contabilidade Gerencial. In: ENCONTRO NACIONAL DE PROGRAMAS DE PÓS-GRADUAÇÃO EM ADMINISTRAÇÃO, 33, 2009, São Paulo, Anais... São Paulo: ANPAD, 2009.

ALTMAN, E I. Financial Ratios, Discriminant Analysis and Prediction of Corporate Bankruptcy. Journal of Finance, v.23, p.589-610, 1968. 
ANTHONY, R.N.; GOVINDARAJAN, V. Sistemas de Controle Gerencial. São Paulo: McGraw-Hill, 2008.

BAILEY, C. D.; BROWN, L. D.; COCCO, A. F. The Effects of Monetary Incentives on Worker Learning and Performance in an Assembly Task. Journal of Management Accounting Research, n.10, p.119-131, 1998.

BEATTY, R.P; ZAJAC, E.J. Managerial Incentives, Monitoring, and Risk Bearing: A Study of Executive Compensation, Ownership, and Board Structure in Initial Public Offerings. Administrative Science Quarterly, v.39, n.2, p.313-335, jun. 1994. Disponível em: http: www:// jstor.org/stable/2393238. Acesso em: 02 fev. 2010.

BEAVER, W. H. Financial Ratios as Predictors of Failure. Journal of Accounting Research, v.4, p.71-1, 1966.

BAKER, G; JENSEN, M.C; MURPHY, K. J. Compensation and Incentives: Practice vs. Theory. The Journal of Finance, v.43, n.3, p.593-616, 1988.

BANKER, R. D.; DATAR, S. M. Sensitivity, Precision And Linear Aggregation of Signals for Performance Evaluation. Journal of Accounting Research, v.27, p.21-39, 1989.

BASU, S. The Conservatism Principle and the Asymmetric Timeliness of Earnings. Journal of Accounting and Economics, v.24, n.1, p.3-37, 1997.

BERGAMINI JR. S. Controles Internos como um Instrumento de Governança Corporativa. Revista do BNDES, v.12, n.24, p.149-188, dez. 2005.

BERLE, A. A.; MEANS, G. C. The modern Corporation and Private Property. New York: MacMillan, 1932.

BONNER, S. E.; SPRINKLE, G. B. The Effects of Monetary Incentives on Effort and Task Performance: Theories, Evidence, and a Framework for Research. Accounting, Organizations, and Society, v.27, p.303-345, 2002.

CAMARGOS, M. A.; HELAL, D. H.; BOAS, A. P. Análise Empírica da Relação entre a Remuneração de Executivos e o Desempenho Financeiro de Empresas Brasileiras. In: XXVII ENCONTRO NACIONAL DE ENGENHARIA DE PRODUÇÃO, 2007, Foz do Iguaçu. Anais... Rio de Janeiro: ABEPRO, 2007. 
CARDOSO, R. L; MARIO, P. C.; AQUINO, A. C. B. Contabilidade Gerencial: Mensuração, Monitoramento e Incentivos. São Paulo, Atlas, 2007.

COOPER, D. R.; SCHINDLER, P. S. Métodos de Pesquisa em Administração. São Paulo: Bookman, 2003.

COSENZA, J. P; ALEGRÍA, A. I. Z; LAURENCEL, L. C. Análise dos Problemas de Agência nas Práticas de Preços de Transferência das Empresas Brasileiras. In: INTERNATIONAL ASSOCIATION FOR ACCOUNTING EDUCATION AND RESEARCH (IAAER) - ASSOCIAÇÃO NACIONAL DE PÓS-GRADUAÇÃO EM CIÊNCIAS CONTÁBEIS (ANPCONT), 3, 2009, São Paulo. Anais... São Paulo: ANPCONT, 2009.

CHOW, C. W.; KATO, Y.; MERCHANT, K. The Use of Organizational Controls and Their Effects on Data Manipulation and Management Myopia: A Japan vs U.S. Comparison. Accounting, Organizations and Society, v.21, n.2/3, p.175-192, 1996.

DALMACIO, F. Z.; REZENDE, A. J.; SLOMSKI, V. Análise Setorial das Medidas de Performance Utilizadas nos Contraltos de Remuneração dos Gestores. Revista Universo Contábil, v.5, n.3, p.06-23, jul./set, 2009.

DAVILA, A.; FOSTER, G. Management Control Systems in Early-Stage Startup Companies. Accounting Review, v.82, n.4, p.907-937, 2007.

DELOACH, J. W. Administração Corporativa de Risco: Estratégia para Relacionar Risco e Oportunidade. New York: Pearson Educação, 2001.

DIKOLLI, S. S. Agent Employment Horizons and Contracting Demand for Forward-Looking Performance Measures. Journal of Accounting Research, v.39, n.3, p.481-493, 2001.

DIKOLLI, S. S.; VAYSMAN, I. Contracting on the Stock Price and Forward-Looking Performance. European Accounting Review, v.15, n.4, p.445-464, 2006.

DUARTE JR., A. M. Risco: Definições, Tipos, Medição e Recomendações para seu Gerenciamento. In: LEMGRUBER, E. et al (Org.) Gestão de Riscos e Derivativos: Aplicação no Brasil. São Paulo: Atlas, 2001.

FELTHAN, G. A.; XIE, J. Performance Measure Congruity and Diversity In Multi-Task Principal/Agent Relations. The Accounting Review, v.69, n.3, p.429-453, 1994. 
FESSLER, N. J. Experimental Evidence on the Links Among Monetary Incentives, Task Attractivess, and Task Performance. Journal of Management Accounting Research, n.15, p.161-176, 2003.

GIETZMANN, M. Introduction to Agency Theory in Management Accounting. Management Accounting, 2. ed, Prentice Hall, 1995.

GITMAN, L. J. Princípios de Administração Financeira. 7. ed. São Paulo: Harbra, 1997. HAIR JR., J. F.; BLACK, W.C.; BABIN, B.J.; ANDERSON, R.E.; TATHAM, R.L. Multivariate Data Analysis. 6. ed. New Jersey: Prentice Hall, 2005.

HENDRINKSEN, E. S; VAN BREDA, M.F. Teoria da Contabilidade. São Paulo: Atlas, 1999.

HEMMER, T. On the Design and Choice of Modern Management Accounting Measures. Journal of Management Accounting Research, v.8, p.87-116, 1996.

HIRST, M. The Controllability of Financial Outcomes. Abacus, v.19, n.1, p.29-38, 1983.

HORNGREN, C. T.; SUNDEM, G. L.; STRATTON, W. Contabilidade Gerencial. 12 ed. São Paulo: Pearson Education do Brasil, 2004.

INDJEJIKIAN, R.; NANDA, D. Dynamic Incentives and Responsibility Accounting. Journal of Accounting and Economics, v.27, p.177-201, 1999.

JENSEN, M. C.; MECKLING, W.H. Theory of The Firm: Managerial Behavior, Agency Costs and Ownership Structure. Journal of Financial Economics, v.3, n.4, p.305-360, oct. 1976.

; MURPHY, K. J.; WRUCK, E.G. Remuneration: Where We've Been, How We Got to Here, What are the Problems, and How to Fix Them. 2004. Social Science Research Network, July. 2004. Disponível em: http://www.ssrn.com/abstract=561305. Acesso em: 02 fev. 2010.

JORION, P. Value at Risk: The New Benchmark for Controlling Derivatives Risk. New York: McGraw Hill, 1997.

KNIGHT, F. Risk, Uncertainty and Profit. Boston: Houghton, 1921.

LAMBERT, R. A. Contracting Theory and Accounting. Journal of Accounting and Economics, v.32, p.3-87, jan 2001. 
LEROY, S.F.; SINGELL JR, L.D. Knight on Risk and Uncertainty. Journal of Political Economy, v.5 n.2, p.394-406, 1987.

MELUMAD, N.; MOOKHERJEE, D.; REICHELSTEIN, S. A Theory of Responsibility Centers. Journal of Accounting and Economics, v.15, p.445-484, 1992.

MERCHANT, K. A. The Effects of Financial Controls on Data Manipulation and Management Myopia. Accounting, Organizations and Society, n.15, p.297-313, 1990.

MERCHANT, K. A.; VAN DER STEDE, W. A.; ZHENG, L. Disciplinary Constraints on the Advancement of Knowledge: The Case of Organizational Incentive Systems. Accounting, Organizations and Society, n.28, p.251-286, 2003.

MILLER, J. S; WISEMAN, R. M; GOMEZ-MEJIA, L. R. The Fit Between CEO Compensation Design and Firm Risk. Academy of Management Journal, v.45, n.4, p.745-756, 2002.

MURPHY, K. Executive Compensation. Handbook of Labor Economics, Orley Ashenfelter and Davids Card, eds. North Holland, p.2485-2563, 1999.

OYADOMARI, J. C. T.; CARDOSO, R. L.; MENDONÇA O. R.; LIMA, M. P. Fatores que Influenciam a Adoção de Artefatos de Controle Gerencial nas Empresas Brasileiras: Um Estudo Exploratório sob s Ótica da Teoria Neo-Institucional. In: $8^{\circ}$ CONGRESSO USP DE CONTABILIDADE E CONTROLADORIA, 2008, São Paulo. Anais... São Paulo, USP, 2008.

ROSS, S. A.; WESTERFIELD, R. W.; JAFFE, J. Corporate Finance. São Paulo: Atlas, 1995.

SAUNDERS, A. Administração de Instituições Financeiras. 2. ed. São Paulo: Atlas, 2000.

TEIXEIRA, A. J. C.; GONZAGA, R. P.; SANTOS, A. V. S. M. A Utilização de ferramentas de Contabilidade gerencial nas empresas do Estado do Espírito Santo. Brazilian Business Review, v.8, n.3, p.108-127, set 2011.

VAN DER STEDE, W. A. The Relationship Between Two Consequences of Budgetary Controls: Budgetary Slack Creation and Managerial Short-Term Orientation. Accounting, Organizations and Society, v.25, p.609-622, 2000.

WERLANG, S. R. C. Simonsen, Inflação, Expectativas Racionais e os Pós-Keynesianos. Revista Brasileira de Economia, n.52, Especial, p.161-166, fev.1998. 
ZMIJEWSKI, M.E. Methodological Issues Related to the Estimation of Financial Distress Prediction Models. Journal of Accounting Research, v.22, p.59-82, 1984.

\section{APÊNDICE 1 - EMPRESAS PARTICIPANTES DA PESQUISA}

\begin{tabular}{|c|c|c|c|}
\hline ID & Empresa & ID & Empresa \\
\hline 1 & ACTA Engenharia Ltda & 71 & Latina \\
\hline 2 & Andrade S/A & 72 & Lorenge \\
\hline 3 & Aracruz & 73 & Luz e Força Santa Maria S/A \\
\hline 4 & Arara Azul & 74 & Metalosa \\
\hline 5 & Arcelor & 75 & Nibrasco \\
\hline 6 & AST Com. Internacional & 76 & Panan \\
\hline 7 & Atacado São Paulo & 77 & Paranasa \\
\hline 8 & Atlantica Automoveis & 78 & Parma Comercial Ltda \\
\hline 9 & Banco do Brasil & 79 & Pemagran \\
\hline 10 & Banco Mercantil & 80 & Perfilados \\
\hline 11 & Bandes & 81 & Plantão Serviços \\
\hline 12 & Banestes & 82 & Podium \\
\hline 13 & Banestes Seguros & 83 & Politintas \\
\hline 14 & BR Distribuidora & 84 & Poltex \\
\hline 15 & Brametal & 85 & Portocel \\
\hline 16 & Brasil Export & 86 & Proimport \\
\hline 17 & Buaiz & 87 & Prosegur \\
\hline 18 & Buteri & 88 & Quimetal Distribuidora \\
\hline 19 & Cafénorte & 89 & Quimetal S/A \\
\hline 20 & Cajugran & 90 & RDG \\
\hline 21 & Casa Sta Terezinha & 91 & Real Café \\
\hline 22 & CBF & 92 & Refrigerantes Coroa \\
\hline 23 & Cesan & 93 & Rima \\
\hline 24 & Cheim Transportes & 94 & Rodosol \\
\hline 25 & CLAC & 95 & Samarco Mineração \\
\hline 26 & Codesa & 96 & Selita \\
\hline 27 & Concrevit & 97 & Serdel \\
\hline 28 & Construtora Aterpa & 98 & Siderurgica Ibiraçu \\
\hline
\end{tabular}




\begin{tabular}{|c|c|c|c|}
\hline 29 & Contek Engenharia & 99 & Silotec \\
\hline 30 & Cooabriel & 100 & Sipolatti \\
\hline 31 & Coopeavi & 101 & Sol Coqueria \\
\hline 32 & Correios & 102 & Solesa \\
\hline 33 & Cotia & 103 & Supermercado Casagrande \\
\hline 34 & CPVV & 104 & Supermercado Santo Antônio \\
\hline 35 & Custódio Farzza & 105 & Tangará \\
\hline 36 & CVC & 106 & Tenax \\
\hline 37 & Decolores & 107 & Terca \\
\hline 38 & Diaço & 108 & Terra Nova Import \& Export \\
\hline 39 & Eisa & 109 & Thork Trading \\
\hline 40 & Eletromil & 110 & Tracomal Mineração \\
\hline 41 & Elkem & 111 & Transcampo \\
\hline 42 & Elson's Produtos Alimentícios Ltda & 112 & Transfinal \\
\hline 43 & Eluma & 113 & Trieste Veículos \\
\hline 44 & Embali & 114 & Tristão \\
\hline 45 & Escelsa & 115 & Trop \\
\hline 46 & Eximbiz & 116 & TV Gazeta \\
\hline 47 & Extrabom & 117 & TV Vitória \\
\hline 48 & Famex & 118 & Unicafé \\
\hline 49 & Fertilizantes Heringer & 119 & Unimed Norte \\
\hline 50 & Fiesa & 120 & Unimed Sul \\
\hline 51 & Fortlev & 121 & Unimed Vitória \\
\hline 52 & Frisa & 122 & Usina Paineiras \\
\hline 53 & Garoto & 123 & Vale S/A \\
\hline 54 & Gazeta S/A & 124 & Venac \\
\hline 55 & Gecel & 125 & Veneza \\
\hline 56 & Golden Distribuidora & 126 & Vessa \\
\hline 57 & Granvitur & 127 & Viação Águia Branca \\
\hline 58 & GS Internacional & 128 & Viação Flexa Branca \\
\hline 59 & Hiper Export & 129 & Viação Grande Vitória \\
\hline 60 & Hispanobrás & 130 & Viação Praia Sol \\
\hline 61 & Hortifruti & 131 & Viação Tabuazeiro \\
\hline 62 & Hospital Meridional & 132 & Vigserv \\
\hline
\end{tabular}




\begin{tabular}{|l|l|r|l|}
\hline 63 & Hospital Metropolitano & 133 & Viminas \\
\hline 64 & Hospital Santa Rita & 134 & Visel \\
\hline 65 & Itabrasco & 135 & Vitoria Apart \\
\hline 66 & Julio Simões & 136 & Vitória Diesel \\
\hline 67 & Kifrango & 137 & Vitória Motors \\
\hline 68 & Kobrasco & 138 & Vitoriawagen \\
\hline 69 & Kurumá & 139 & Vix Logística \\
\hline 70 & Lasa & 140 & White Martins \\
\hline
\end{tabular}

